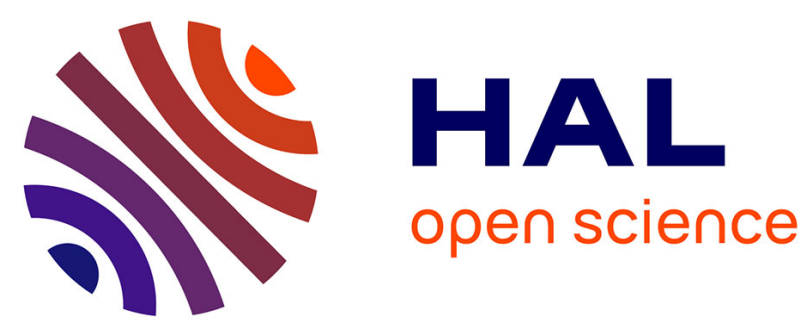

\title{
Dynamic Workflow in Grid-MAS Integration Context
} Paola Salle, Frédéric Duvert, Danièle Hérin, Stefano A. Cerri

\section{To cite this version:}

Paola Salle, Frédéric Duvert, Danièle Hérin, Stefano A. Cerri. Dynamic Workflow in Grid-MAS Integration Context. SYNASC 2007 - 9th International Symposium on Symbolic and Numeric Algorithms for Scientific Computing, Sep 2007, Timisoara, Romania. 10.1109/SYNASC.2007.68 . lirmm00192508

\section{HAL Id: lirmm-00192508 https://hal-lirmm.ccsd.cnrs.fr/lirmm-00192508}

Submitted on 28 Nov 2007

HAL is a multi-disciplinary open access archive for the deposit and dissemination of scientific research documents, whether they are published or not. The documents may come from teaching and research institutions in France or abroad, or from public or private research centers.
L'archive ouverte pluridisciplinaire HAL, est destinée au dépôt et à la diffusion de documents scientifiques de niveau recherche, publiés ou non, émanant des établissements d'enseignement et de recherche français ou étrangers, des laboratoires publics ou privés. 


\section{Dynamic Workflow in Grid-MAS Integration Context}

\author{
Paola Salle \\ LIRMM : University Montpellier 2 \& CNRS \\ 161 Rue Ada, \\ 34392 Montpellier Cedex 5, France \\ salle@lirmm.fr \\ Daniele Herin \\ LIRMM : University Montpellier 2 \& CNRS \\ 161 Rue Ada, \\ 34392 Montpellier Cedex 5, France \\ herin@lirmm.fr
}

\begin{abstract}
This paper addresses the architectural foundations of $d y$ namic workflows in distributed multi-agent systems (MAS) integrated in Grid context. The purpose is to design an architecture at the same time taking into consideration tasks dependencies among agents, adaptation with respect to historic lessons learnt from past behaviour (memory) and the autonomous decisions when an unpredicted event occurs. In order to do this, given one ontology, called AGIO, which describes Agent-Grid Integration, we propose a workflow based on MAS with a complementary decision-making aid using Markov Logic Networks (MLN).
\end{abstract}

\section{Introduction}

Workflows are required in several domains especially in distributed systems. In the current literature, we can appreciate many different versions of workflows (e.g: workflow mining, workflow based on MAS, workflow ontology, etc.) but they are usually criticised by the users because they are considered as too rigid in their structure and in their mechanism. Indeed, when an unpredicted event occurs at run time, it is not easy to take into account this event in the task's sequence. In accordance with the tradition of Agent technologies, we know that Agents are different from Objects in the sense that their decisions are autonomous and dynamic and not predefined or static, i.e. they depend on the flow of "real" events and not just on "forecasted" ones. While today's Multi-Agent Systems have improved their performances as adequate models of multi-centric intelli-

\author{
Frederic Duvert \\ LIA : University Avignon \\ 339, chemin des Meinajaries, \\ 84911 Avignon Cedex 9 \\ frederic.duvert@univ-avignon.fr \\ Stefano A. Cerri \\ LIRMM : University Montpellier 2 \& CNRS \\ 161 Rue Ada, \\ 34392 Montpellier Cedex 5, France \\ cerri@lirmm.fr
}

gence, they have not yet fully convinced the "real applicative world". This has been extensively documented for instance by [4]. These authors have in fact called for an "integration" between MAS and Grid services which goes beyond a simple synergy for mutual help, or interoperability, as shown in most of the traditional literature.

It should be noted that this full integration has indeed been achieved in its essence by recent work [3, 7]. With the advent of Agent-Grid Integration, it is necessary to consider workflows in this context since Grid provides power and a support for sharing resources in different virtual communities. We need to describe the basics of workflow. Ontology may be defined as being a formalisation of the concepts, properties and relationships in a given domain[5]. We have therefore extended the ontological description of Agents and Grid services as presented in [3]. Since this ontology (AGIO) enables us to formalize our new workflow and integrate it in this context. The way of "how" to use the past in order to forecast the future and thus offering "optimal bayesian weights" to potential decisions on the next most promising move to make, is represented by Markov Logic Networks [12].

We capitalized on these models for our reflections about an innovative architecture (and prototypical infrastructure) for dynamic workflows seen as history-aware and adaptive Grid services. We argue that a secure, reliable, open and standard architecture / infrastructure offered by Grid services is at the moment the best baseline supporting workflow technologies acceptable for real applications. At the same time the adoption of concepts from MAS facilitates the conception of flexible multi-centric intelligent systems that are able to model autonomous human and artificial agent's organizations cooperating for a common shared business. 


\section{Main Concepts of Workflow}

"Workflow is the automation of a process (partial or complete), during which documents, information and tasks pass from a participant to another within a working group and in conformity with a set of rules. A system of workflow defines, creates and manages the execution of such processes" [6].

We define a workflow as a set of Activities. By Activity, we mean the process activated by an Agent when performing a Task constrained by some Constraining relations in a local evaluation Environment. Tasks are "statically" described actions.

\section{Dynamic Workflow}

In order to model realistic workflows, where both artificial and human Agents coexist and offer services to each other, communicate and work together, we need to be able to design an architecture that in some way facilitates the dynamic generation and choice of actions according to the real flow of events at run time and the adaptation to the past (experiential learning). Therefore our goal is to define the primitives enabling such a dynamic workflow model. We can then apply the model to a simple yet significant example of a realistic workflow. In the following, we present a very synthetic summary of our Ontology for Workflows which is an extension of AGIO and the innovative architecture for a dynamic workflow.

\subsection{Ontology}

Dynamic workflows and autonomous agents are not just an interesting evolution of current technologies, but rather belong to the fundamental priorities if one wishes to model realistically collaborative activities of human and artificial agents engaged on the Net. The workflow, presented hereafter, aims to be as dynamic as possible -choices are generated when needed- and moves (actions) are supposed to be chosen by agents considered as autonomous as possible

In this case, workflows are considered as a Grid service with all the advantages that procure Grid and the notion of Service. For our workflow, we take the same principle of STROBE (Stream Object Environment), model of communication, proposed and detailed by [8]. Therefore, we add these concepts in AGIO taking into account our definitions about workflows : Plan, Activity, Task, Constraint, Stream and Environment. We link them by the concept of "Agent". A representation with this structure enables a workflow more dynamic than other ones based on Petri-nets [1]. When an unpredicted event occurs, agents are to be able to reorganize their future Activities without defining again an entire workflow. We think that an Agent manager is required to keep the global coherence of the workflow, thanks to the ontology. In the next section, we present this manager and a complementary decision-making aid using MLN for agents. In fact, it enables to consider uncertain situation and it provides a powerful tool in such event.

\subsection{Workflow Agent}

In our proposition, we are situated in a multi-agent system where each agent has a knowledge of his domain which is partial but there is an Agent Manager which has a global knowledge of the application's domain and it advises agent's activities. It defines, with the users, the goal and the sequence of tasks. We consider that the most important is to perform a goal defined with the users rather than to respect the time constraints. The manager advises the other agents which participate in the sequence of tasks. This view point is proposed by [13]. When an Agent, participating in the workflow, can't accomplish a Task, its manager has some possibilities to modify the situation by using different tools (e.g : send a message or modify the task's priority). This manager keeps the global coherence of workflow since he knows the set of Activities and the goal to perform.

\subsection{Workflow with Markov Logic Net- works}

MLN are a combination of logic -representing the domain semantics- and Markov networks both identifying a reasoning based on probabilistic logic [12]. [10, 11] express logic formulas used and give very good definitions about their use.

Based on a training set of knowledge facts, MLN infer the probability of future events concerning the current situation. The aim of the MLN is to query predicates' validation. According to the data provided, a training step is necessary to give a weight to each logic formula. The grounded predicates (formulas) are instances of representation objects. The weights are fixed on the formula of the Knowledge Base (KB). A test step is to query a formula in a KB.

Knowledge Base can be used as a representation of workflow Activities. According to Activities accomplished and a goal defined, we can question the KB for a future Activity which enables us to realize our goal. If we query the probability that $\mathrm{A}$ occurs and if $\mathrm{C}$ appears then we can decide which solutions are more appropriated.

Using MLN in Workflow contribute to help choosing an Activity, taking into account the probability of success, calculated by the markov networks with the training historical test. The inference mechanism is generalized to any formula in the MLN. The probability of the formula $F_{1}$, given that the formula $F_{2}$, is provided by: $P\left(F_{1} \mid F_{2}, L, C\right)=$ 
$\frac{\sum_{x \in \mathcal{X}_{F_{1}} \cap \mathcal{X}_{F_{2}}} P\left(X=x \mid M_{L, C}\right)}{\sum_{x \in \mathcal{X}_{F_{1}}} P\left(X=x \mid M_{L, C}\right)}$ with $L$ is an MLN, $C$ is a set of constants appearing in $F_{1}$ and $F_{2}$ and $\mathcal{X}_{F_{i}}$ is the set of worlds where $F_{i}$ appears. For example, in a First Order Logic(FOL) Knowledge Base, we describe the relations between members of a university, professor, students, their different graduation, and the research papers they have submitted. With a training set of data concerning these people, the MLN system learns the weights to put in each FOL formula. In the test phasis, we may request, for example, the MLN the probability that a student A is advised by a professor knowing the fact that they have written the same paper together.

\section{Example}

Our scenario is an administrative university workflow. A student's association would like to organize a sport event. Workflow can provide a support and a guideline on the different step in order to accomplish successfully their project. Thus students will have advises from workflow manager to organize the event, by using the workflow, i.e. they must perform several Tasks in order to obtain funds :

- A1 : ask the Chair the permission for the event

- A2 : ask for funds

- A3 : obtain the authorization

- A4 : obtain funds

We describe this example in figure 1. Notice that this representation is a static illustration at time $\mathrm{T}$ of the dynamic workflow : the representation will be different at time $\mathrm{T}+$ 1 and we recall that in this paper, we does not take into account constraints on time.

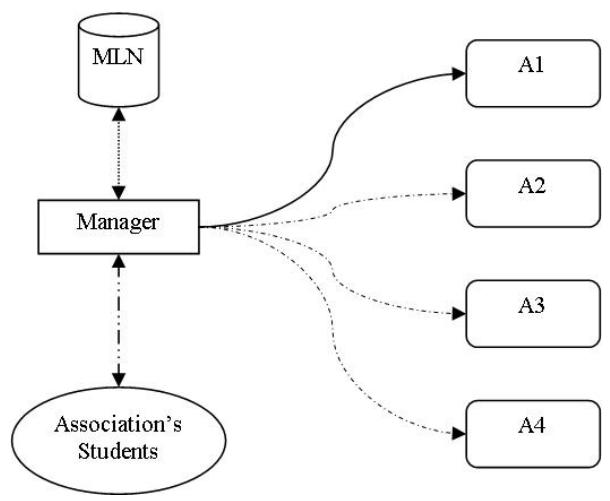

Figure 1. Example of the workflow

First, the manager agent discusses with the students to define a goal to perfom. According to its global domain knowledge, the manager agent defines a preliminary sequence, and launches the workflow by sending a request to the first activity. If there is no response, the manager agent sends a request to the MLN to know "what is the probability to perform the goal defined if there is not this activity". Then it can report the current activity or modify the next activities sequence. If the activity is accomplished then the manager agent chooses an another activity necessary to perform the goal.

In the choice context of the optimal Activity, the workflow manager can use some heurisitics and use MLN. Using a strong First-Order Logic base, will help the manager to respect the global system coherence. Moreover using MLN enables the manager to request the system on some occuring events. It will have an idea of the future learning from the past experience. It has Activities history and can request for the " most effective task", or the probability of success at time T, using MLN and its Alchemy program [9] helping in picking an Activity. The Markov Logic Network uses a knowledge base, describing relations and predicates modelling the student association project scenario.

Based on some the logs of these tasks, Table 1 represents a few results from a partial solution for this problem. We want to predict probabilities of the success of human projects. For instance, the fact that an association does not ask for funds does not imply that the aim will not succeed. But an association must ask for authorization from the Chair in order to hold an event on the campus. These results have been found using the Alchemy program.

\begin{tabular}{|l|l|}
\hline Type of tasks done & $\begin{array}{l}\text { Probability to } \\
\text { realise Project }\end{array}$ \\
\hline \hline notR(authorization $),$ notO $($ funds $)$ & 0.001 \\
\hline$R($ authorization $)$, notO $($ funds $)$ & 0.58 \\
\hline$R($ authorization $)$, (funds $)$ & 0.93 \\
\hline
\end{tabular}

Table 1. Example of MLNs usages for the workflow service, $R$ is for Request $(X), O$ is for $\operatorname{Obtain}(X)$

Having these results, the workflow manager can infer its knowledge and consequently choose the Activity. Then it can evaluate the first element of the promised list to activate at time $\mathrm{T}+1$. It iterates these operations again and again at each time, thus dynamically choosing the next activity to do.

\section{Discussion}

In this paper, we have presented the basic elements for a dynamic workflow created as a Web-service in a Grid environment. We have focused our point of view on the choice of tasks by the user : important elements for the 
dynamicity of workflow. We did not focus our work on the management of resources nor any temporal constraints. Creating a dynamic workflow has already been proposed using Markov Decision Process in [2]. This model uses Bayesian and Markov Networks to support a strategy in order to produce a "universal plan". A policy assignes to each state of the world, an action that is expected to be optimal over the period of consideration. Their approach is to "automatically establish the workflow logic" and because they do not logically or semantically formalize the workflow, incoherencies and inconsistencies can appear. MLNs use Markov networks with a combination of probabilistic logic that enhances workflow robustness. This formalization brings a semantic level and rigorous way to describe the workflow. Consequently it may not be possible to have false instantiations of tasks.

\section{Conclusion and perspectives}

The issue of dynamic workflows coincides with dynamic planning in Artificial Intelligence: from past experience this is a very challenging problem that can not be solved by a simplistic proposal. However, we hope to have set the stage for a significant progression, and in a reasonably short time, in order to address simply and effectively two of the major problems that dynamic workflows pose. These are:

1. the run-time evaluation of the next move to do in a distributed, multicentric environment of autonomous Agents cooperating on the Grid, and

2. the experiential learning of the likelihood of success of each candidate "move", in order to choose the most promising one at each turn.

Their integration within one of the most recent and acceptable proposals for a MAS-Grid service unified representation has allowed us to identify the kernel of an Ontology for Dynamic Workflows compatible with the Agent Grid Integration Ontology previously developed. Finally, an extremely simple easily understood example of workflow in a "government" process (students and the administration of a University organizing a student's event) has been used as a mock-up scenario for validating the proposed key concepts. Our next challenges will be:

1. to evaluate the quality of the service in realistic scenarios

2. to optimize both the performances of the run time support for the workflow service and of the conceptual description necessary for designing / simulating tasks, activities, constraints and the conversation flow among autonomous Agents in order to fully exploit the value added by our Ontology, at both define time as well as at run time.

\section{References}

[1] W. Aalst. Three Good reasons for Using a Petri-netbased Workflow Management System. In S. Navathe and T. Wakayama, editors, Proceedings of the International Working Conference on Information and Process Integration in Enterprises (IPIC'96), pages 179-201, Camebridge, Massachusetts, 1996.

[2] P. Doshi, R. Goodwin, R. Akkiraju, and K. Verma. Dynamic workflow composition using markov decision processes. In ICWS '04: Proceedings of the IEEE International Conference on Web Services, page 576, Washington, DC, USA, 2004. IEEE Computer Society.

[3] F. Duvert, C. Jonquet, P. Dugnie, and S. A. Cerri. AgentGrid Integration Ontology. In R. Meersman, Z. Tari, and P. Herrero, editors, AWeSOMe'06 : International Workshop on Agents, Web Services and Ontologies Merging,, pages 136-146. Springer, volume 4277 of LNCS, November 2006.

[4] I. Foster, N. R. Jennings, and C. Kesselman. Brain meets brawn: Why grid and agents need each other. In $A A M A S$ '04: Proceedings of the Third International Joint Conference on Autonomous Agents and Multiagent Systems, pages 8-15, Washington, DC, USA, 2004. IEEE Computer Society.

[5] T. R. Gruber. A translation approach to portable ontologies. Knowledge Acquisition, 5(2):199-220, June 1993.

[6] D. Hollingsworth. Wfmc: Workflow reference model. Specification, Workflow Management Coalition, 1995. TC001003.

[7] C. Jonquet. Dynamic Service Generation: Agent interactions for service exchange on the Grid. $\mathrm{PhD}$ thesis, University Montpellier 2, Montpellier, France, November 2006.

[8] C. Jonquet and S. A. Cerri. The STROBE model: Dynamic Service Generation on the Grid. Applied Artificial Intelligence, Special issue on Learning Grid Services, 19(910):967-1013, October-November 2005

[9] S. Kok, P. Singla, M. Richardson, and P. Domingos. The alchemy system for statistical relational ai. Technical report, University of Washington, Seattle, WA, 2005. $\mathrm{http} / / /$ alchemy.cs.washington.edu.

[10] N. J. Nilsson. Principles of artificial intelligence. Morgan Kaufmann Publishers Inc., San Francisco, CA, USA, 1980.

[11] N. J. Nilsson. Probabilistic logic. Artificial Intelligence, 28(1):71-88, 1986.

[12] M. Richardson and P. Domingos. Markov logic networks. Machine Learning, 62(1-2):107-136, 2006.

[13] P. Rongier, A. Liegeois, and O. Simonin. Markovian analysis of a heterogeneous system: application to acooperation task for multiple consumer robots. In Systems, Man, and Cybernetics, 2000 IEEE International Conference on, volume 4, pages 3033-3038. IEEE Computer Society, 2000. 